\title{
Oxidative Damage to DNA: Counter Ion-Assisted Addition of Water to Ionized DNA
}

\section{Supporting Material}

Robert N. Barnett ${ }^{\dagger}$, Angelo Bongiorno ${ }^{\dagger}$, Charles L. Cleveland ${ }^{\dagger}$, Abraham Joy $^{\neq}$, Uzi Landman ${ }^{\dagger}$, Gary B. Schuster ${ }^{\neq} *$

Schools of Physics ${ }^{\dagger}$ and Chemistry \& Biochemistry ${ }^{\neq}$

Georgia Institute of Technology

Atlanta, GA 30332 


\section{S1. The QM/MM method}

Atomic-scale modeling and simulations of biological phenomena often involves large systems embedded in complex environments. ${ }^{1,2}$ While a quantum-mechanical (QM) treatment of the full system is prohibitive because of it's large size, classical schemes based on molecular mechanical (MM) force fields are routinely employed to address such large systems. ${ }^{1}$ However, MM schemes cannot account for bond breaking or charge redistribution processes occurring, for instance, in the course of enzymatic reactions in proteins. ${ }^{3}$ As a compromise, the description of such processes is enabled by the formulation and implementation of hybrid methods, where quantum and molecular mechanical schemes (QM/MM) are appropriately combined. ${ }^{3,4}$ Various hybrid schemes have been proposed to date, ${ }^{4-14}$ with the main issue pertaining to merging of the QM and MM schemes in a seamless manner, thus allowing reliable and stable simulations of systems of biological relevance. In this study we developed and used a QM/MM scheme based on a plane-wave density-functional approach. Our QM/MM scheme allows for a consistent description of the electrostatics at the boundary between the QM and $\mathrm{MM}$ regions.

Our system consists of a 14 base-pair B-DNA oligomer [d(5'AAGGAAGGAAGGAA-3')]/[d(3'-TTCCTTCCTTCCTT-5')] with the sugar-phosphate groups, $28 \mathrm{Na}^{+}$counter ions and a bath of 804 water molecules The region described by our quantum mechanical (QM) approach consists of $230 \mathrm{QM}$ atoms, including the two central GC base pairs of the tetradecamere olignucleotide and associated sugar phosphate groups, $4 \mathrm{Na}^{+}$counter ions, $32 \mathrm{H}_{2} \mathrm{O}$ molecules, 4 "bridge atoms" (see below). Our quantum mechanical scheme is based on a plane-wave density-functional approach, 
formulated for finite system calculations. ${ }^{15}$ The exchange and correlation energy is accounted for within a generalized gradient approximation (GGA). ${ }^{16}$ Only valence electrons are described explicitly with the use of norm conserving pseudopotentials ${ }^{17}$ for the core-valence interactions. There are 352 occupied wave-functions of each spin (when using local spin-density functional, LSD, calculations). In the classical (MM) region the Amber 96 potentials ${ }^{18,19}$ were used.

In the following, we focus our discussion on the coupling between the QM and MM regions. First, we reiterate the basic elements of our QM/MM system. The DNA molecule and it's hydrating environment (including the $\mathrm{Na}^{+}$counter ions) are divided into two types of regions, a molecular mechanical (MM) region where the interactions and forces between the atoms are described through prescribed interatomic potentials (here we use the Amber force field), and a quantum mechanical (QM) region where the electrons are treated quantum mechanically (here we use density functional theory, in conjunction with pseudopotentials and a plane-wave basis) and the forces on the ions are evaluated through the use of the Hellman-Feynman theorem (in addition to the direct interionic repulsion).

The QM and MM regions are coupled. Thus, the atoms in the MM region carry partial charges and they interact with the ions in the QM region as well as with the quantum-mechanically evaluated electronic charge density, and similarly the coulomb potentials due to the partial charges of the atoms in the MM region act as external potentials on the electrons(and ions) in the $\mathrm{QM}$ region. In our case the QM region is embedded (see Fig. 2 in the text of the paper) between two MM regions (one on the 5'side and the other on the 3'-end ). The boundaries between the MM regions and the QM 
region are defined by two "linking atoms" on each strand of the DNA duplex; as described below, the linking atoms exhibit a dual $\mathrm{MM} / \mathrm{QM}$ nature. On the 5'side the linking atom is chosen to be $\mathrm{C}^{\prime}$ (which is bonded to the $\mathrm{C} 4$ ' atom of the QM region) and on the $3^{\prime}$ end the linking atom is $\mathrm{C} 4$ ' (which is bonded to the $\mathrm{C} 5$ ' of the $\mathrm{QM}$ region); see Figure S1. To properly terminate the QM region (i.e. saturate the bonds of the QM carbon atoms at the interface with the $\mathrm{MM}$ region, i.e. the $\mathrm{C} 4{ }^{\prime}$ and $\mathrm{C} 5^{\prime}$ on the $5^{\prime}$ - and $3^{\prime}$-ends respectively), the linking atoms are described through the use of $\mathrm{H}$-like local pseudopotential. ${ }^{8}$ We wish to preserve the partial charges assigned to the linking atoms by the MM scheme; in the following we denote these charges as $\mathrm{Q}^{\mathrm{MM}}\left(\mathrm{C}^{\prime}\right)=0.16 \mathrm{e}$ and $\mathrm{Q}^{\mathrm{MM}}\left(\mathrm{C}^{\prime}\right)=.-0.07 \mathrm{e}$ (the assigned values are those given by the Amber parameterization). Together with the above mentioned hydrogen-like bond-saturation, this leads us to use in the construction of the one-electron local norm-conserving pseudopotentials of the C5' and C4" linking atoms, nuclear charges of $\mathrm{q}(\mathrm{C} 5 ")=1+\mathrm{Q}^{\mathrm{MM}}(\mathrm{C} 5 ")$ and $\mathrm{q}(\mathrm{C} 4 ")=1+$ $\mathrm{Q}^{\mathrm{MM}}(\mathrm{C} 4 ")$; when added to the (negative) charge of a single electron the assigned $\mathrm{MM}$ partial charges are recovered for each of the linking atoms.

To maintain the MM values for the bond-length and vibrational frequency of the bond connecting each linking atom with the corresponding neighboring QM carbon atom, we construct (using density functional theory) a parameterized harmonic spring interaction potential between the linking atom (described by the aforementioned pseudopotential) and the neighboring (QM) carbon atom, with the other bonds made by the latter saturated by hydrogens (this serves to model the saturated $\mathrm{sp}^{3}$ hybridization of the these carbons). For each atomic configuration the force corresponding to this harmonic potential is used in order to cancel the force component along the bond 
obtained from the quantum mechanical calculation. Subsequently, in molecular dynamics simulations, or in structural relaxations, the interactions between the linking atoms and the neighboring carbon atoms in the $\mathrm{QM}$ region are calculated using the $\mathrm{MM}$ harmonic potentials.

The interaction between the QM region and non-bonded MM ions includes van der Waals ${ }^{18,19}$ and coulomb interactions. Spill-out effects ${ }^{20}$ and spurious large electrostatic interactions at the interface between the QM and MM regions are avoided by gaussian smearing the MM effective ionic charges. For each element, the width of the gaussian is assigned according to its $\mathrm{MM}$ effective charge. A simple linear relation between the excess charge of the atom and the width of the gaussian is derived from DFT calculations on charged atoms. 


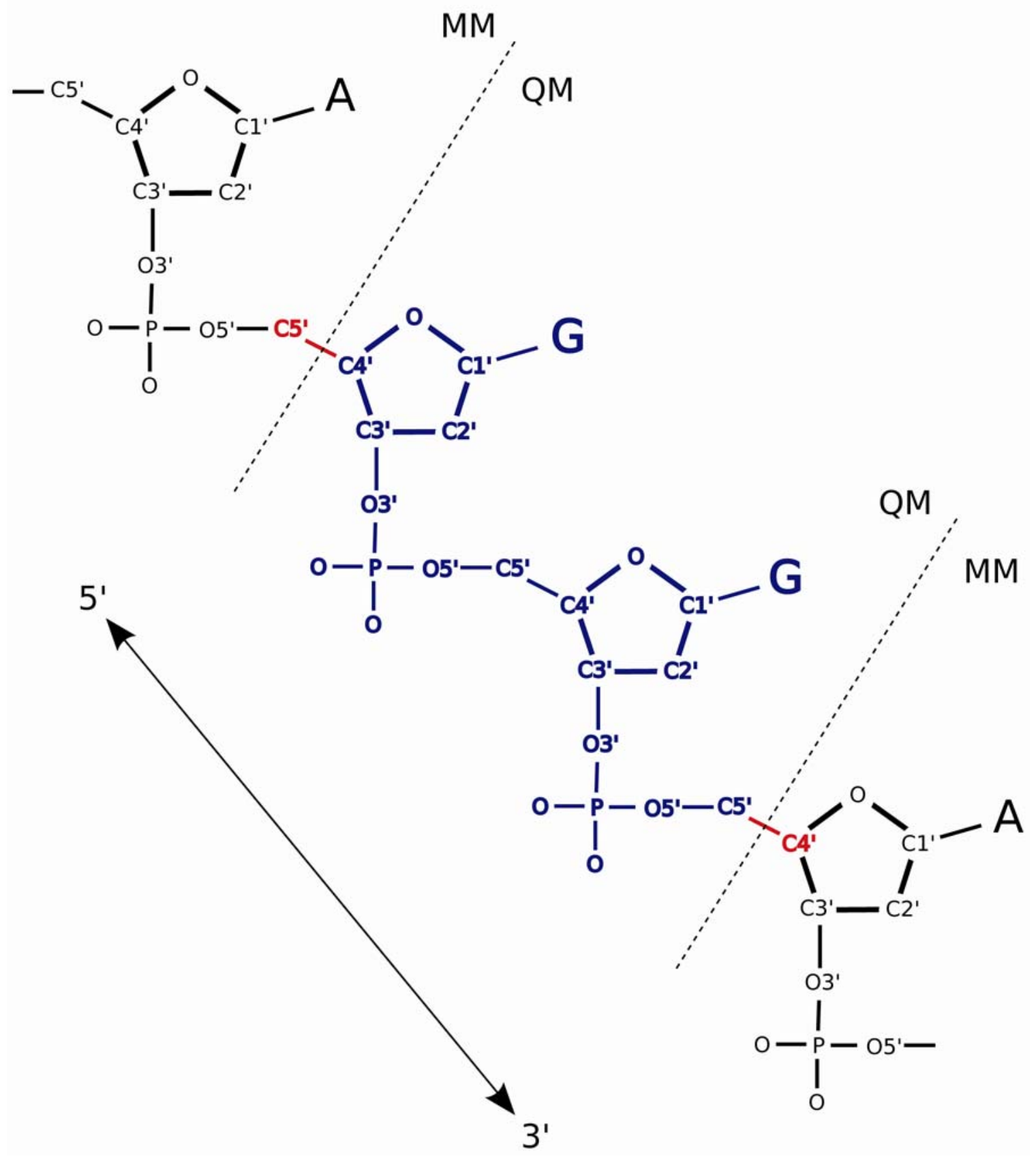

Figure S1: Definition of the $\mathrm{QM}$ and $\mathrm{MM}$ regions on the strand containing the GG step. The atoms in blue belong to the QM region, and those in red are the bridging atoms. 


\section{References (S1)}

1. M. Karplus and G.A. Petsko, Nature 347, 631 (1990).

2. R.N. Barnett, C.L. Cleveland, A. Joy, U. Landman, and G.B. Schuster, Science 294, 567 (2001).

3. A. Warshel and M. Levitt, J. Mo. Biol. 103, 227 (1976).

4. M.J. Field, P.A. Bash, and M. Karplus, J. Comp. Chem. 11, 700 (1990).

5. X. Assfeld and J.-L. Rivail, Chem. Phys. Lett. 263, 100 (1996).

6. D. Bakowies and W. Thiel, J. Phys. Chem. 100, 10580 (1996).

7. J. Gao, P. Amara, C. Alhambra, and M.J. Field, J. Phys. Chem. A 102, 4714 (1998).

8. Y. Zhang, T.-S. Lee, and W. Yang, J. Chem. Phys. 110, 46 (1999).

9. M. Eichinger, P. Tavan, J. Hutter, and M. Parrinello, J. Chem. Phys. 110, 10452 (1999).

10. I. Antes and W. Thiel, J. Phys. Chem. A 103, 9290 (1999).

11. N. Reuter, A. Dejaegrere, B. Maigret, and M. Karplus, J. Phys. Chem. A 104, $1720(2000)$.

12. V. Kairys and J. H. Jensen, J. Phys. Chem. A 104, 6656 (2000).

13. D. Das, K.P. Eurenius, E.M. Billings, P. Sherwood, D.C. Chatfield, Milan Hodoscek, and B.R. Brooks, J. Chem. Phys. 117, 10534 (2002).

14. M. Elstner, T. Frauenheim, and S. Suhai, J. Mol. Struct. 632, 29 (2003). 
15. R.N. Barnett and U. Landman, Phys. Rev. B 48, 2081 (1993).

16. J.P. Perdew, K. Burke, and M. Ernzerhof, Phys. Rev. Lett. 77, 3865 (1996).

17. N. Troullier and J.L. Martins, Phys. Rev. B 43, 1993 (1991).

18. D.A. Case et. al.,. Amber 6 (University of California, San Francisco, 1999).

19. P.A. Kollman, R. Dixon, W. Cornell, T. Fox, C. Chipot, and A. Pohorille, Computer Simulations of Biomolecular Systems; (Elsevier: Amsterdam, 1997); Vol.

3.

20. A. Laio, J. Vande Vondele, and U. Rothlisberger, J. Chem. Phys. 116, 6941 (2002). 


\section{S2. Spatial distribution of the electron-hole}

In Table S2 we give the fraction of the hole in different regions of the QM subsystem Here a region is defined as the union of spheres with a radius of $4 \mathrm{a}_{0}\left(\mathrm{a}_{0}\right.$ is the Bohr radius) centered on each of the atoms (excluding hydrogen) defining the region. No double counting occurs within a given region, but can occur when two regions intersect.

Table S2: Fraction of hole in regions

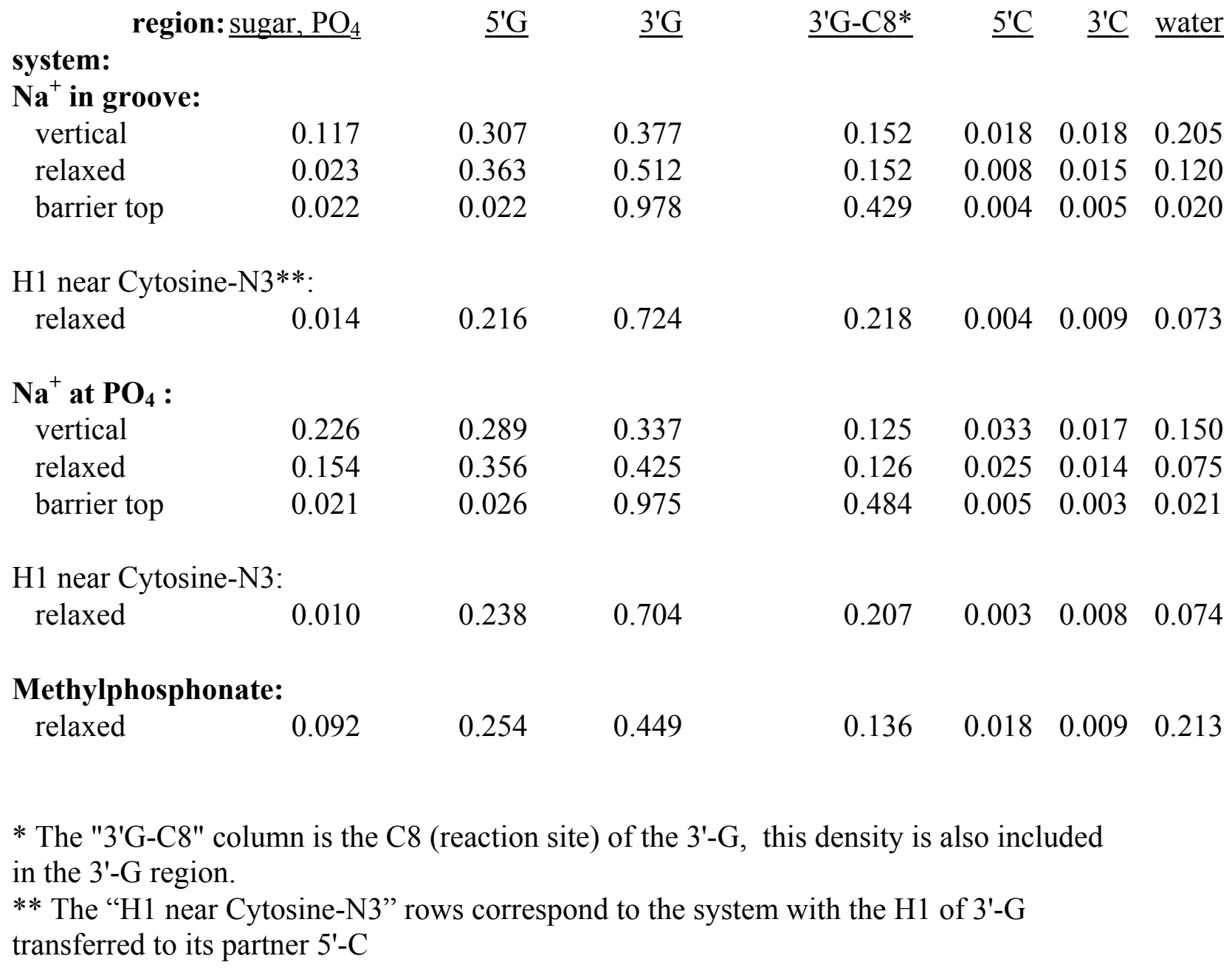




\section{S3. Interatomic distance variations along reaction paths.}

\section{Table S3.}

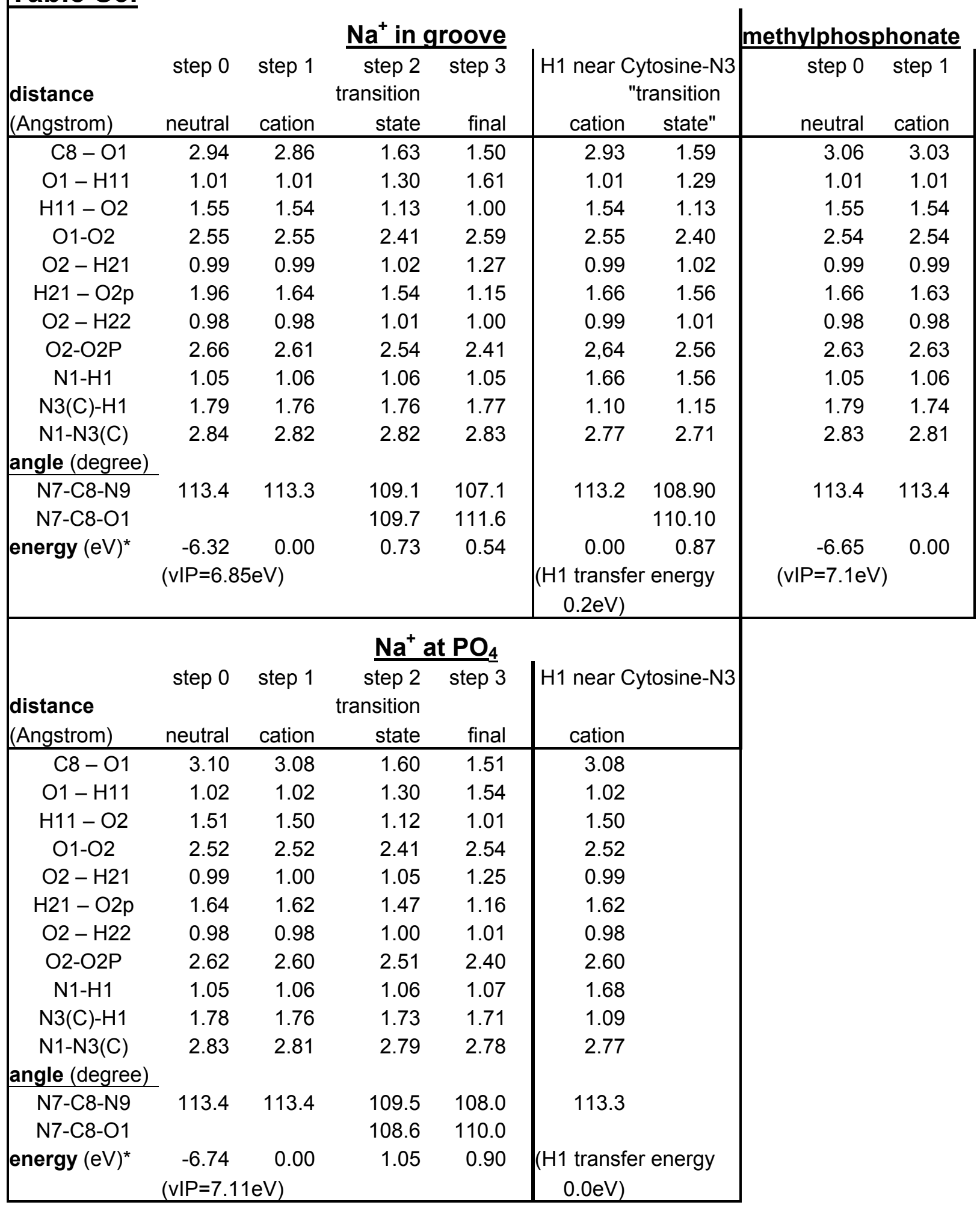


*The energies are with reference to tha of the relaxed DNA radical cation, taken as zero. The adiabatic ionization potential (aIP) is given by the absolute value of the enrgy of the relaxed neutral, and the relaxation energy is the difference vIP-aIP.

Information in the Table for the transition state (Step 2) and final state (Step 3) are given for those cases where a transition state (saddle point) could be determined (see also Fig. 5 in the main text).

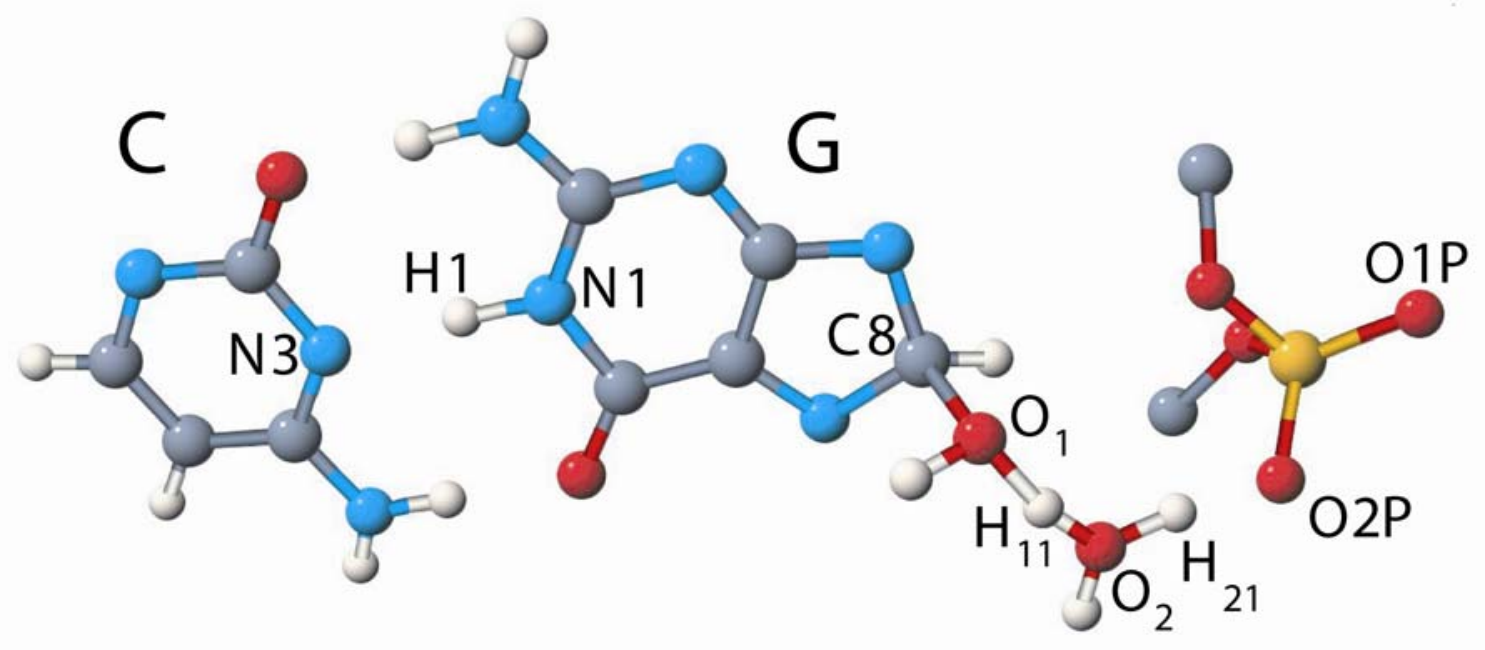

Figure S3a: The part of the system participating directly in the reaction. The labels define the interatomic distances given in Table S3. 


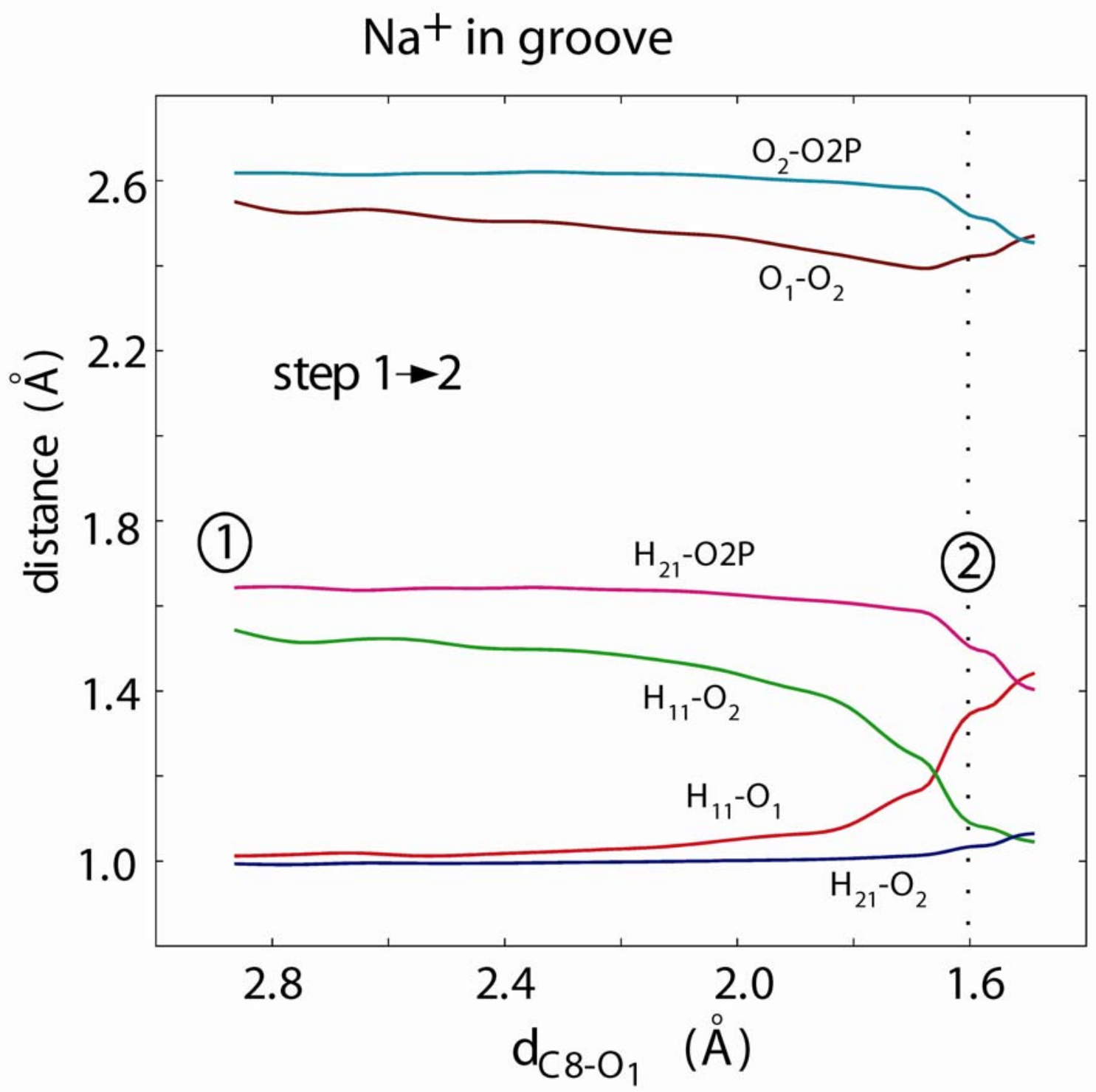

Figure S3b: Variation of interatomic distances plotted versus the reaction coordinate, dC8-O1, starting from the initial configuration (step 1) and ending slightly past the transition state (step 2). The transition state is denoted by a vertical dotted line. The results correspond to the reaction with the counter ion located in the groove, and the $\mathrm{H} 1$ near the 3'-G. 


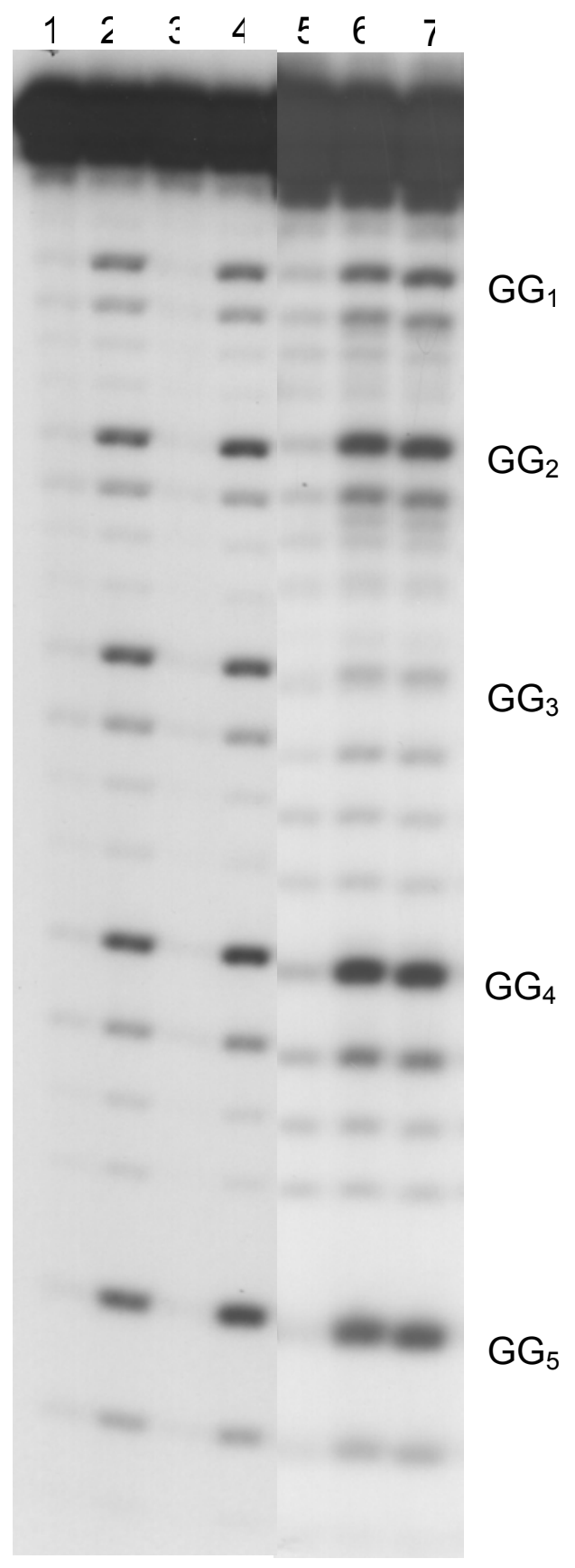

Figure S4: Autoradiograph of PAGE gel following irradiation and Fpg treatment of AQDNA. Positions of substitution refer to Figure 6 of the paper.

Lanes 1-2: AQ-DNA $\left\{\mathrm{a}, \mathrm{b}, \mathrm{c}, \mathrm{d}=\mathrm{PO}_{4}{ }^{-}\right\} 0$ and 7 min irradiation.

Lanes 3-4: AQ-DNA $\left\{\mathrm{a}, \mathrm{b}, \mathrm{c}=\mathrm{PO}_{3}\left(\mathrm{CH}_{3}\right), \mathrm{d}=\mathrm{PO}_{4}^{-}\right\} 0$ and 7 min irradiation. Lanes 5-7: AQ-DNA $\left\{\mathrm{a}, \mathrm{b}, \mathrm{c}=\mathrm{PO}_{4}^{-}, \mathrm{d}=\mathrm{PO}_{3}\left(\mathrm{CH}_{3}\right)\right\} 0,7$ and 10 min irradiation. 